\title{
Article
}

\section{Real-Space Image of Charged Patches in Tunable-Size Nanocrystals}

\author{
Jordi Martínez-Esaín 1,2, Ana Pérez-Rodríguez ${ }^{2}$, Jordi Faraudo ${ }^{2, *} \mathbb{C}$, Esther Barrena ${ }^{2}$, Ramón Yáñez ${ }^{1}$, \\ Carmen $\mathrm{Ocal}^{2}$ and Susagna Ricart ${ }^{2, *}$
}

1 Departament de Química, Universitat Autònoma de Barcelona (UAB), 08193 Barcelona, Spain; martinezesain.jordi@protonmail.com (J.M.-E.); ramon.yanez@uab.cat (R.Y.)

2 Institut de Ciència de Materials de Barcelona, ICMAB-CSI C, Campus de la UAB, 08193 Barcelona, Spain; ana.perez.rodriguez.c@gmail.com (A.P.-R.); ebarrena@icmab.es (E.B.); cocal@icmab.es (C.O.)

* Correspondence: jfaraudo@icmab.es (J.F.); ricart@icmab.es (S.R.)

check for

updates

Citation: Martínez-Esaín, J.;

Pérez-Rodríguez, A.; Faraudo, J.; Barrena, E.; Yáñez, R.; Ocal, C.; Ricart, S. Real-Space Image of Charged Patches in Tunable-Size Nanocrystals. Materials 2022, 15, 1455. https:// doi.org/10.3390/ma15041455

Academic Editors: Elena Taran, P. Davide Cozzoli, Anders Barlow and Tian Zheng

Received: 24 November 2021 Accepted: 11 February 2022 Published: 15 February 2022

Publisher's Note: MDPI stays neutral with regard to jurisdictional claims in published maps and institutional affiliations.

Copyright: (c) 2022 by the authors. Licensee MDPI, Basel, Switzerland. This article is an open access article distributed under the terms and conditions of the Creative Commons Attribution (CC BY) license (https:// creativecommons.org/licenses/by/ $4.0 /)$.

\begin{abstract}
The remarkable dual nature of faceted-charge patchy metal fluoride nanocrystals arises from the spontaneous selective coordination of anionic and cationic ligands on the different facets of the nanocrystals. In previous studies, the identification and origin of the charge at the patches were obtained by combining computer simulations with indirect experimental evidence. Taking a step further, we report herein the first direct real-space identification by Kelvin probe force microscopy of the predicted faceted-charge patchy behavior, allowing the image of the dual faceted-charge surfaces. High-resolution transmission electron microscopy reveals the detailed nanocrystal faceting and allows unambiguously inferring the hydrophilic or hydrophobic role of each facet from the identification of the surface atoms exposed at the respective crystallographic planes. The success of the study lies in a foresighted synthesis methodology designed to tune the nanocrystal size to be suitable for microscopy studies and demanding applications.
\end{abstract}

Keywords: patchy nanocrystals; charged patches; HRTEM; KPFM

\section{Introduction}

Currently, there is substantial activity on the development and fabrication of particles with nanopatterned surfaces, i.e., particles with chemically or topographically distinct surface domains (patches) [1]. One of the reasons for this interest is their use as building blocks in the self-assembly of complex hierarchical structures, with the patches providing localized interaction sites promoting self-assembly [2-6]. Efficient methods for the generation of micrometer -and sub-micrometer-sized patchy colloids have already been developed. Currently, the efforts are directed toward the search for patchy nanocrystals (NCs), with sizes of the order of tens of nanometers [1,2]. For example, Choueiri et al. obtained discrete patches of various types of polymers over gold NCs [1]. The origin of the patches in that case was the thermodynamic segregation of the different polymers. Recently, we obtained patchy $\mathrm{LnF}_{3} \mathrm{NCs}$ (Ln: La, Ce, or Pr) with patches exhibiting different electric charges [7]. As shown schematically in Figure 1a, the charge duality arises from the selective functionalization of the diverse NC patches with anionic and cationic ligands. The underlying mechanism, identified by molecular dynamics simulations, is due to the spontaneous faceting of hexagonal $\mathrm{LnF}_{3} \mathrm{NCs}$, which leads to planes with different affinities for different kinds of ligands.

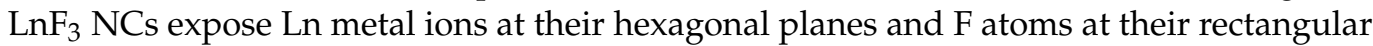
planes. In general, ligands containing carboxylate moieties coordinate with metal atoms (X-type coordination following the covalent bond classification for nanoscale systems) [8,9], while nitrogen-based molecules interact with fluorine atoms (electrostatic interaction based on H-bonding $(\mathrm{F} \cdots \mathrm{H}-\mathrm{N})$. The hexagonal surfaces of $\mathrm{LnF}_{3} \mathrm{NCs}$ are stabilized by citrate and acetate anions coordinated to Ln, acquiring a negative charge, and the rectangular surfaces are stabilized by ammonium cations, acquiring a positive charge. This effect is illustrated in Figure $1 b$ as seen in MD simulations. Experimentally, we confirmed the presence of 
adsorbed ligands (citrate, acetate, and ammonium) in $\mathrm{LnF}_{3} \mathrm{NC}$ s by combining ${ }^{1} \mathrm{H}-\mathrm{NMR}$ and IR characterization. Therefore, each facet results spontaneously functionalized and charged in a selective way by the ligands present during the synthesis.

a

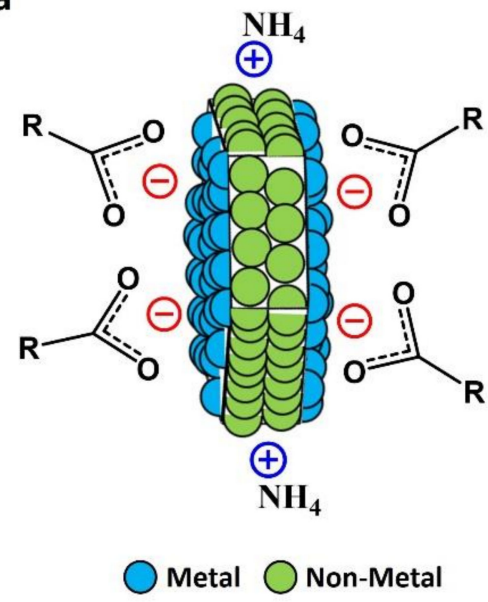

b

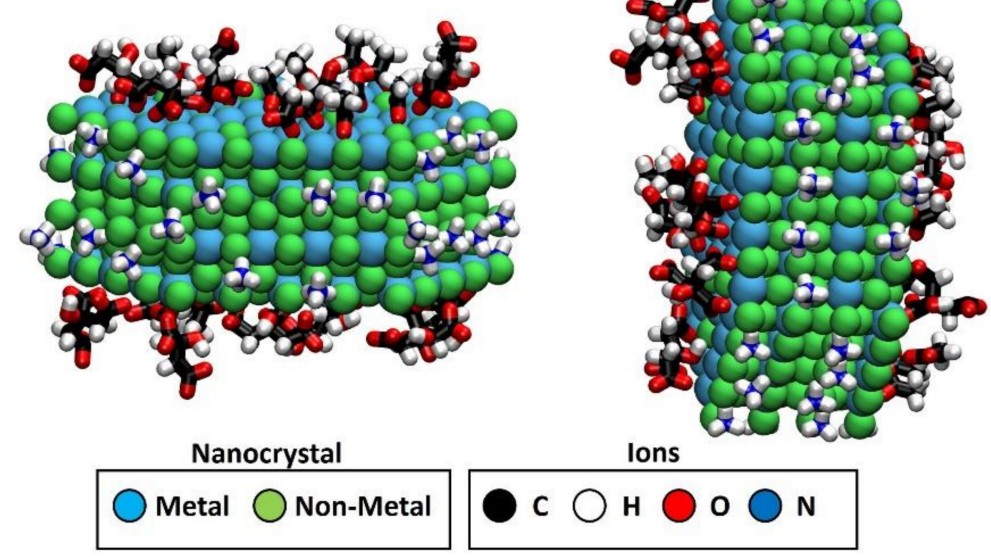

Figure 1. (a) Schematic representation of faceted-charge patchy NC with the local charges in the exposed patches due to the ionic stabilizers; (b) snapshot (two different views) generated from the

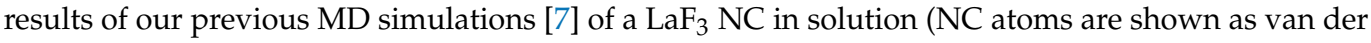
Waals spheres, and adsorbed ions are shown in licorice representation; water and non-adsorbed ions are not shown for simplicity). The images show adsorbed acetate and citrate anions (found only at the hexagonal face) and adsorbed ammonium cations (found only at the rectangular face), thereby predicting the formation of charged patches due to ligand adsorption. These adsorptions gave a charge density of approximately $-3.4 \mathrm{e} / \mathrm{nm}^{2}$ for hexagonal facets and $+3.4 \mathrm{e} / \mathrm{nm}^{2}$ for the rectangular ones, obtaining a global charge density of $-0.47 \mathrm{e} / \mathrm{nm}^{2}$ considering the NC. Images in (b) were generated with Visual Molecular Dynamics software (VMD) version 1.9.3 (Urbana-Champaign, IL, USA) [10].

Unfortunately, the experimental techniques mentioned above do not have spatial resolution, and they cannot detect the proposed patchy distribution of ligands predicted by MD simulations. This limitation motivated the present study.

As in other examples of patchy NCs, the main challenge to obtain a detailed characterization of the patches comes from the nanometric size and location of these patches over the NC surface [1,11-13]. Hence, the development of methods providing a direct and reliable picture of the patches of NCs and their nature is of fundamental importance for a reproducible and rational synthesis of patchy nanocrystals. In the above-discussed example [7], the small size of the $\mathrm{LnF}_{3}$ particles $(<10 \mathrm{~nm})$ impeded a direct characterization of the charges of the individual patches. In this work, we present a strategy designed to overcome this limitation, providing a real-space characterization of $\mathrm{LnF}_{3}$ patchy nanocrystals and, in particular, their local electrical charge, morphology, and dimensions.

We divided the work in two parts. First, we present the details of improved synthetic methods of patchy $\mathrm{LnF}_{3} \mathrm{NCs}$ capable of obtaining a full control over particle size. After exploring diverse strategies, a method for obtaining a tunable range of NC sizes from 5 to $\sim 15 \mathrm{~nm}$ is presented. This step is essential to obtain NCs of optimal size with large enough patches suitable for direct characterization by microscopy techniques. Indeed, control of NCs size is a highly desirable feature also for NCs applications in demanding fields where size restrictions are decisive factors (e.g., medical applications) [14-17]. In the second part, we provide a thorough characterization of optimal size NCs via highresolution transmission electron microscopy (HRTEM) and Kelvin probe force microscopy (KPFM). We explicitly show the presence of patches with opposite charges at the surface of the nanoparticles. 
As far as we know, this is the first reported instance of a complete visualization of these patchy systems, in which the patches themselves and their charge are unveiled.

\section{Materials and Methods}

\subsection{Materials}

Lanthanum(III) acetate hydrate $99.9 \%$, cerium(III) acetate hydrate $99.9 \%$, citric acid $99 \%$, tetramethylammonium hydroxide $25 \mathrm{wt} . \%$ in water, and ammonium fluoride $>99.99 \%$ were purchased from Sigma-Aldrich (Madrid, Spain). Acetone $99.5 \%$ was purchased from Scharlau (Barcelona, Spain). All reagents were used as received without further purification.

\subsection{Nanocrystal Synthesis}

Coprecipitation method. In a $50 \mathrm{~mL}$ round-bottom flask equipped with a condenser and a magnetic stirrer, citric acid $(2.25 \mathrm{mmol})$ in $16 \mathrm{~mL}$ of MilliQ water was neutralized with tetramethylammonium hydroxide $(6.75 \mathrm{mmol})$, followed by the addition of $\mathrm{Ln}\left(\mathrm{CH}_{3} \mathrm{COO}\right)_{3} \cdot \mathrm{H}_{2} \mathrm{O}(1.5 \mathrm{mmol})$. The initial solution was maintained at room temperature, heated until $100{ }^{\circ} \mathrm{C}$ or introduced in an ultrasonic bath, and then $\mathrm{NH}_{4} \mathrm{~F}(4.5 \mathrm{mmol})$ in $4 \mathrm{~mL}$ of MilliQ water was injected dropwise. After $2 \mathrm{~h}$ of reaction, the final mixture was allowed to reach room temperature depending on the conditions. $\mathrm{LnF}_{3}$ particles were separated from the reaction medium by the addition of $10 \mathrm{~mL}$ of acetone, followed by centrifugation at 10,000 rpm for $20 \mathrm{~min}$. Separated NCs were redispersed in $20 \mathrm{~mL}$ of MilliQ water or methanol, forming stable colloidal dispersions.

Hydrothermal post-treatment. Final washed colloidal suspensions in water were added into a Teflon vessel, and then the reactor was sealed; with vigorous stirring, the solution was heated up at 170 or $210^{\circ} \mathrm{C}$ for $2 \mathrm{~h}$. Final solutions were washed with acetone at 10,000 rpm for $20 \mathrm{~min}$. Separated NCs were redispersed in $20 \mathrm{~mL}$ of MilliQ water or methanol, forming stable colloidal dispersions.

Hydrothermal method. In a Teflon vessel, citric acid $(2.25 \mathrm{mmol})$ in $16 \mathrm{~mL}$ of MilliQ water was neutralized with tetramethylammonium hydroxide $(6.75 \mathrm{mmol})$, followed by the addition of $\mathrm{Ln}\left(\mathrm{CH}_{3} \mathrm{COO}\right)_{3} \cdot \mathrm{H}_{2} \mathrm{O}(1.5 \mathrm{mmol})$. Then, $\mathrm{NH}_{4} \mathrm{~F}(4.5 \mathrm{mmol})$ in $4 \mathrm{~mL}$ of MilliQ water was injected dropwise. After the addition, reactor was sealed and heated up at $210{ }^{\circ} \mathrm{C}$. After $2 \mathrm{~h}$ of reaction, the final mixture was allowed to reach room temperature. $\mathrm{LnF}_{3}$ particles were separated from the reaction medium by the addition of $10 \mathrm{~mL}$ of acetone, followed by centrifugation at 10,000 rpm for $20 \mathrm{~min}$. Separated NCs were redispersed in $20 \mathrm{~mL}$ of MilliQ water or methanol, forming stable colloidal dispersions.

Microwave reaction. In a Teflon vessel, citric acid $(2.25 \mathrm{mmol})$ in $16 \mathrm{~mL}$ of MilliQ water was neutralized with tetramethylammonium hydroxide $(6.75 \mathrm{mmol})$, followed by the addition of $\mathrm{Ln}\left(\mathrm{CH}_{3} \mathrm{COO}\right)_{3} \cdot \mathrm{H}_{2} \mathrm{O}(1.5 \mathrm{mmol})$. Then, $\mathrm{NH}_{4} \mathrm{~F}(4.5 \mathrm{mmol})$ in $4 \mathrm{~mL}$ of MilliQ water was injected dropwise. After the addition, the vessel was introduced in a microwave (MW) to be heated up at $200{ }^{\circ} \mathrm{C}$. After 20 min of reaction, $\mathrm{LnF}_{3}$ particles were separated from the reaction medium by the addition of $10 \mathrm{~mL}$ of acetone, followed by centrifugation at 10,000 rpm for $20 \mathrm{~min}$. Separated NCs were redispersed in $20 \mathrm{~mL}$ of MilliQ water or methanol, forming stable colloidal dispersions.

\subsection{Characterization Techniques}

X-ray powder diffraction (XRD) patterns of the samples were recorded using a Phillips XPert diffractometer equipped with a two circle diffractometers and $\mathrm{Cu}$ tube (Malvern Panalytical Ltd., Malvern, UK). Transmission electron microscopy (TEM) micrographs were obtained on a $120 \mathrm{kV}$ JEOL 1210 TEM (JEOL, Freising, Germany), with a resolution point of $3.2 \AA$. High-Resolution transmission electron microscopy (HRTEM) micrographs were obtained on a $200 \mathrm{kV}$ JEOL 2011 TEM (JEOL, Freising, Germany), with a resolution point of $1.8 \AA$ at $200 \mathrm{kV}$. Samples for TEM analysis were prepared by spreading a drop of as-prepared NCs diluted dispersion on amorphous carbon-coated grids and then dried in air. All images have been obtained at Servei de Microscòpia of UAB. A microwave (MW) oven of Milestone model FlexiWAVE (Milestone Srl, Bérgamo, Italy) was used to heat up 
samples at $200{ }^{\circ} \mathrm{C}$. SEM analyses were performed through the Electron Microscopy Service at ICMAB using the QUANTA FEI 200 FEG-ESEM (Hillsboro, OR, USA) with a resolution of $0.8 \mathrm{~nm}$ at $30 \mathrm{kV}$ in high vacuum. Atomic force microscopy (AFM) measurements were carried out at room temperature and ambient conditions using the commercial head and control unit from Nanotec Electrónica (Madrid, Spain). Kelvin probe force microscopy (KPFM) was used in the frequency modulation mode (FM-KPFM), in which the tip is excited by an ac voltage $(\sim 0.5 \mathrm{~V})$ at a frequency $\left(\mathrm{f}_{\mathrm{AC}} \sim 0.7 \mathrm{kHz}\right)$, while a feedback loop adjusts the $\mathrm{DC}$ bias needed to nullify the frequency shift $(\Delta \mathrm{f})$ at $\mathrm{f}_{\mathrm{AC}}$. When scanning over a surface, this methodology provides a surface potential (SP) image, which is directly correlated to the local effective work function $(\varphi)$ and/or to the distribution of electrostatic charges. In our setup, the voltage is applied to the tip such that a higher SP yields a lower $\varphi$. The SP and topographic maps are obtained simultaneously, in a single pass, and confirmed in the lift mode, where the tip scans some tens of nm above the surface. This procedure ensures no crosstalk between signals, while it permits establishing direct correspondence between morphology and electrostatic properties. CrPt-coated Si tips mounted in cantilevers with a nominal spring constant of $\mathrm{k} \approx 3 \mathrm{~N} \cdot \mathrm{m}^{-1}$ (BudgetSensors, Sofia, Bulgaria) were used. Waterdispersed NCs were deposited by drop-casting on commercial Au-coated mica substrates (Georg Albert PVD, Silz, Germany) to obtain well-spread distributions of particles all over the sample surface. These substrates guarantee atomically flat (111) oriented terraces of typically several hundreds of nanometers across separated by monoatomic steps (see Figure S1). Different surface locations were measured for each sample containing NCs using the same tip for at least one set of experiments. All data were analyzed with the WSxM freeware (Version 5.0 Develop 9.4, Madrid, Spain) [18].

\section{Results and Discussion}

\subsection{Tuning the Size of Patchy $L n F_{3} N C s$}

Among the different methods employed to synthesize $\mathrm{LaF}_{3} \mathrm{NC}$ s with a desired (tunable) size, the first selected approach is an extension of the aqueous coprecipitation method (see Section 2 for details) considered in our previous work [7]. The relatively small NCs obtained from the coprecipitation method are enlarged in size by subsequently applying an appropriate treatment (post-treatment).

At room temperature, the aqueous coprecipitation method produces hexagonal crystalline NCs, $\sim 5 \mathrm{~nm}$ in size, with or without ultrasonic bath activation (see TEM images and X-ray diffraction patterns in Figures S2 and S3). At $100{ }^{\circ} \mathrm{C}$, the same method leads to larger hexagonal crystalline NCs with a size of $\sim 7 \mathrm{~nm}$ (see Figure 2a,d). In order to further increase the NC size, we apply a hydrothermal treatment to the NCs obtained at $100{ }^{\circ} \mathrm{C}$. As illustrated in Figure 2, by using $170^{\circ} \mathrm{C}$ and $210^{\circ} \mathrm{C}$ for the subsequent enlargement treatment, the final achieved sizes become $\sim 8 \mathrm{~nm}$ and $\sim 12 \mathrm{~nm}$, respectively. These results are summarized in Table 1. Noticeably, the enlargement of the $\mathrm{LaF}_{3} \mathrm{NCs}$ from 7 to $12 \mathrm{~nm}$ is accompanied by a better-defined hexagonal shape, i.e., the NCs exhibit improved faceting. 

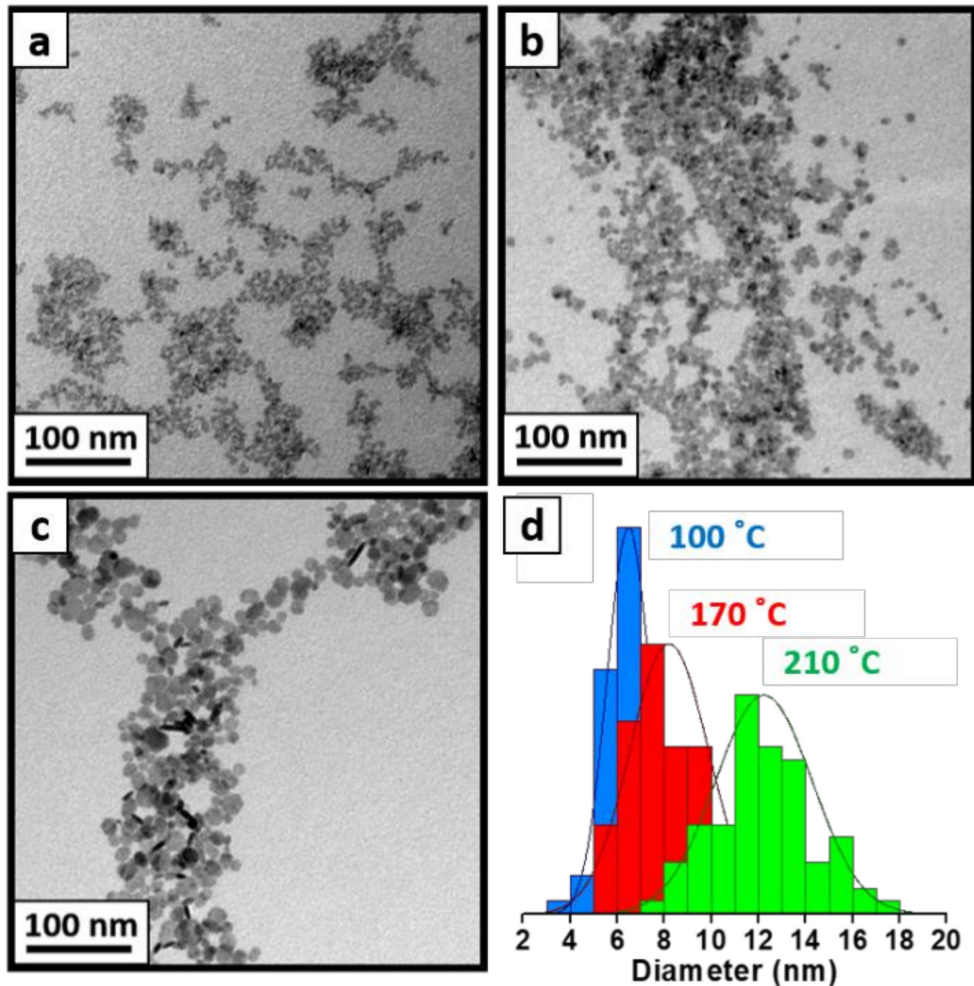

Figure 2. TEM images of as-synthesized $\mathrm{LaF}_{3}$ patchy NCs via coprecipitation method at $100{ }^{\circ} \mathrm{C}(\mathbf{a})$ and after a hydrothermal post-treatment at $170{ }^{\circ} \mathrm{C}(\mathbf{b})$ and $210^{\circ} \mathrm{C}(\mathbf{c})$. TEM histograms (d) of three images are also shown to corroborate their increasing size: as-synthesized $\mathrm{LaF}_{3} \mathrm{NCs}$ (blue), $\mathrm{LaF}_{3}$ after hydrothermal post-treatment at $170{ }^{\circ} \mathrm{C}$ (red), and $\mathrm{LaF}_{3}$ after a hydrothermal post-treatment at $210{ }^{\circ} \mathrm{C}$ for two additional hours (green).

Table 1. Size of all $\mathrm{LaF}_{3}$ and $\mathrm{CeF}_{3} \mathrm{NCs}$ obtained by TEM histograms. The synthesis methods or post-treatments are indicated as follows: Co-Prec., coprecipitation; Hydro., hydrothermal; MW, microwave; rt, room temperature. * Data obtained from [7] for comparison.

\begin{tabular}{cccc}
\hline NCs & 1st Treatment & 2nd Treatment & TEM Size (nm) \\
\hline & Co-Prec. Rt & - & $4.7 \pm 1.4$ \\
Co-Prec. rt & - & $4.7 \pm 1.0$ \\
& sonication & - & $7.0 \pm 1.0$ \\
$\mathrm{LaF}_{3}$ & Co-Prec. $100{ }^{\circ} \mathrm{C}$ & Hydro. $170{ }^{\circ} \mathrm{C}$ & $8.2 \pm 1.7$ \\
& Co-Prec. $100{ }^{\circ} \mathrm{C}$ & Hydro. $210^{\circ} \mathrm{C}$ & $12.2 \pm 2.1$ \\
& Co-Prec. $100{ }^{\circ} \mathrm{C}$ & - & $15.7 \pm 3.5$ \\
& Hydro. $210{ }^{\circ} \mathrm{C}$ & - & $13.0 \pm 3.3$ \\
\hline & MW $200{ }^{\circ} \mathrm{C}$ & - & $7.6 \pm 2.3$ \\
$\mathrm{CeF}_{3}$ & $\mathrm{Co-Prec.} 100{ }^{\circ} \mathrm{C}$ & - & $12.9 \pm 3.3$ \\
& Hydro. $210{ }^{\circ} \mathrm{C}$ & - & $14.2 \pm 3.4$ \\
\hline
\end{tabular}

To gain insight into the effect of the post-treatment temperature used, we also employed direct hydrothermal and microwave reactions at similarly high temperatures. In both cases, hexagonal-faceted $\mathrm{LaF}_{3} \mathrm{NCs}$ with a size of 13-16 nm (Figures S4 and S6) were obtained, with the characteristic crystalline structure (see XRD data in Figures S5 and S7). These results, also summarized in Table 1, indicate that the synthetic methods presented here enable obtaining $\mathrm{LaF}_{3} \mathrm{NCs}$ with a high control over the size in the range of 5-16 nm, making them suitable for the synthesis of NCs for application where the size is a critical factor. The synthetic approximation discussed here has also been applied to produce other 
$\mathrm{LnF}_{3} \mathrm{NCs}$. Table 1 and Figures S4-S8 include the results for hexagonal crystalline $\mathrm{CeF}_{3}$ NCs with sizes ranging from 8 to $14 \mathrm{~nm}$ [7].

In summary, $\mathrm{LaF}_{3}$ and $\mathrm{CeF}_{3} \mathrm{NC}$ of different sizes can be synthesized using diverse aqueous methodologies (Table 1), all of them using the same precursors and with the same resulting crystalline structure.

As shown schematically in Figure $1 \mathrm{a}$, all $\mathrm{LnF}_{3} \mathrm{NCs}$ reported in Table 1 are expected to be stabilized by coordination of surface atoms with ligands initially present in the synthesis medium [7]. In our case, the synthesis medium contained citrate and acetate anions and ammonium and tetramethylammonium cations (see Section 2). According to the mechanism described in Figure 1, we predict that the hexagonal surfaces of $\mathrm{LnF}_{3} \mathrm{NCs}$ are stabilized by citrate and acetate anions coordinated to $\mathrm{La}$ or $\mathrm{Ce}$, acquiring a negative charge, and the rectangular surfaces are stabilized by ammonium cations, acquiring a positive charge (Figure 2a). This prediction is directly tested in the next subsection.

\subsection{Full Imaging of Patchy $\mathrm{LnF}_{3}$ Nanocrystals}

In this subsection, we describe the characterization of patchy NCs, including the imaging of their facets and the identification of differently charged patches. We perform this characterization for the case of the $\mathrm{CeF}_{3} \mathrm{NCs}$ synthesized via hydrothermal treatment at $210{ }^{\circ} \mathrm{C}$ (see Table 1) since they have an optimal size for the techniques employed here.

The particle faceting is clearly seen in the representative HRTEM images of the oriented $\mathrm{CeF}_{3} \mathrm{NCs}$ (Figure 3). In view of the hexagonal face shown in Figure 3b, (004) and (600) planes are correspondingly indexed. This particular configuration is explained thanks to the specific orientations adopted by the NC (flat in Figure 3b, on-edge in Figure 3c) that allow visualizing both facets. However, a closer look at the $\mathrm{NC}$ when oriented through their rectangular face (Figure 3c) reveals that only the (004) oriented plane is clearly distinguished, meaning that the hexagonal exposed face is $\{0001\}$. Thus, the rectangular planes observed in Figure $3 \mathrm{~b}$ must have (600) orientation with an exposed face of the $\{1 \overline{1} 00\}$ family.
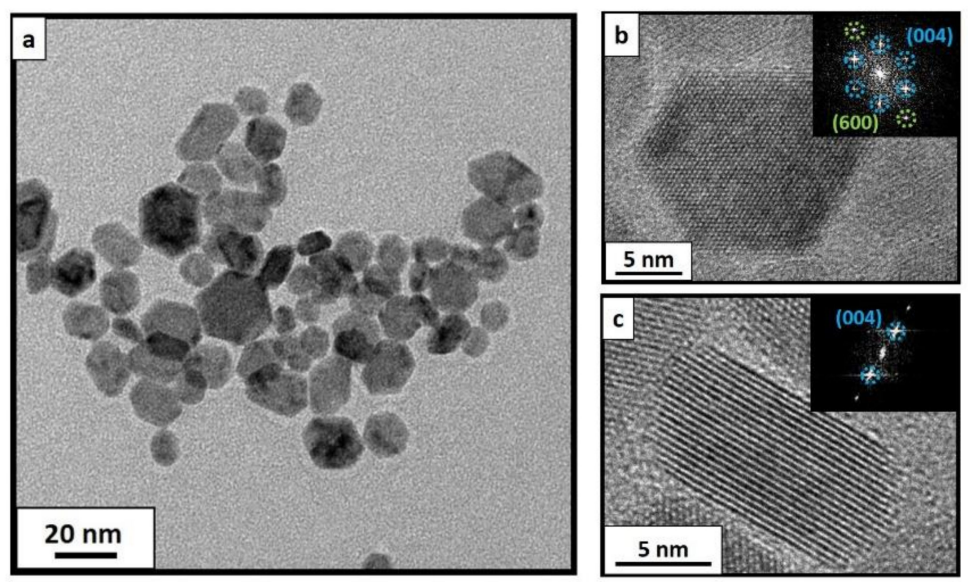

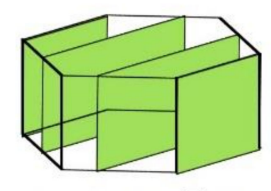

d $\{0001\}$

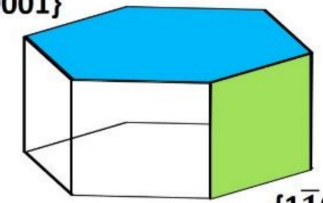

$(600) \rightarrow\{1 \overline{1} 00\}$

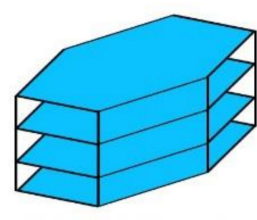

(004) $\rightarrow\{0001\}$

Figure 3. TEM image of $\mathrm{CeF}_{3} \mathrm{NCs}$ synthesized via hydrothermal method at $210{ }^{\circ} \mathrm{C}(\mathbf{a})$. Well faceted-NCs could be observed tilted in several directions, randomly oriented. HRTEM images of as-synthesized $\mathrm{CeF}_{3} \mathrm{NCs}$ via hydrothermal treatment at $210{ }^{\circ} \mathrm{C}$ with their corresponding faceting in $\{1 \overline{1} 00\}$ (b) and in $\{0001\}$ (c) planes. Deduced experimental faceting, as well as the exposed elements in each facet (d).

As commented above, fluorine-rich $\{1 \overline{1} 00\}$ patches would be stabilized by cations, generating a positive global charge. In contrast, $\{0001\}$ hexagonal facets were metal-terminated and, therefore, the attachment of anions onto them would lead to a final local negative charge. To identify the selectively charged patches, we consider how the charges are distributed and where they are located (Figure 1a). Hexagonal $\{0001\}$ faces exposed in flat lying NCs are relatively large and, therefore, easily detected via TEM (see also Figure S4). 
In contrast, visualizing $\{1 \overline{1} 00\}$ planes, exhibiting smaller lateral extension, is challenging unless a high number of particles are lying in the on-edge configuration (i.e., exposing this facet as in the model of Figure 1a).

Due to the adequate ratio between NCs adopting each type of configuration (i.e., exposing one or the other plane), the facet-dependent behavior characteristic of $\mathrm{LnF}_{3} \mathrm{NCs}_{\text {, }}$ can be studied here. As seen by TEM (Figure 4a), the preparation of highly concentrated solutions ( $\sim 150 \mathrm{mM}$ ) of these NCs led to two types of NC organizations: (i) monodisperse NCs lying flat on the grid and exposing their $\{0001\}$ planes, highlighted with red squares, and (ii) rod-like assemblies of NCs exposing $\{1 \overline{1} 00\}$ planes, highlighted with blue squares. These structures are only formed at such high concentrations in solution, allowing the possible creation of analogous ionic bridge interactions [19] between NCs, permitting directional self-assembly through $\{0001\}$ planes. Indeed, the unique on-surface rod-like NCs organization, in which the $\{1 \overline{1} 00\}$ planes are exposed, matches the commented need for having a high number of particles in a perpendicular position to access these facets by imaging techniques.

a

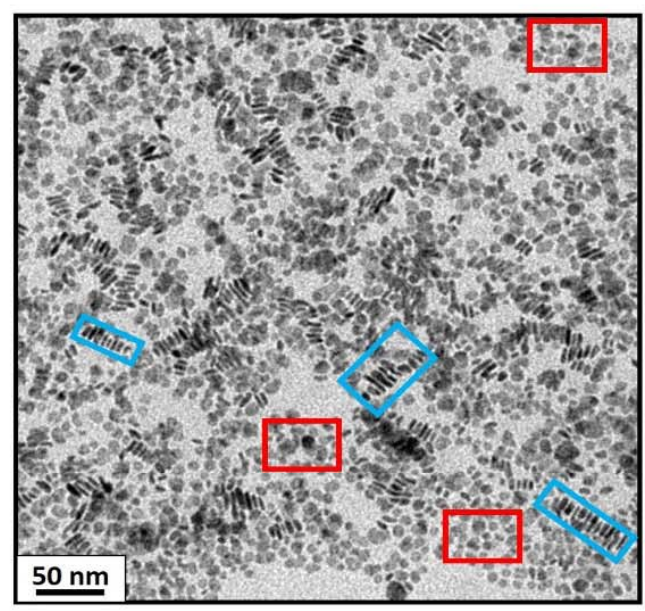

d b

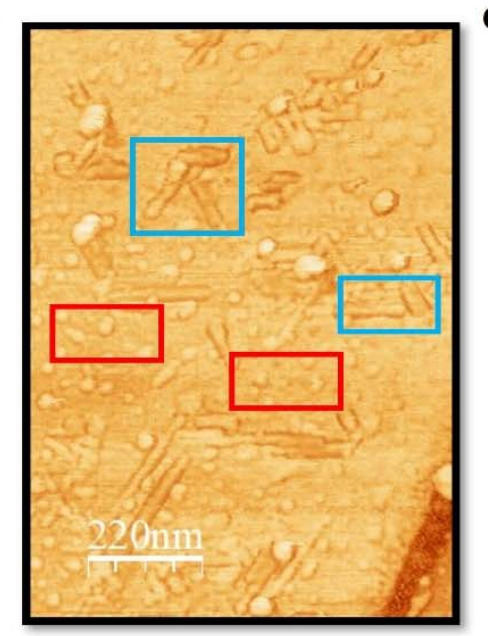

C
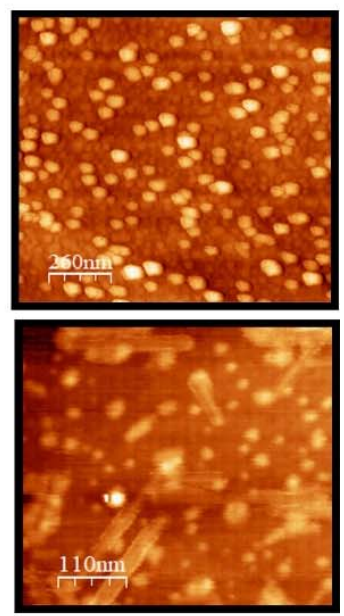

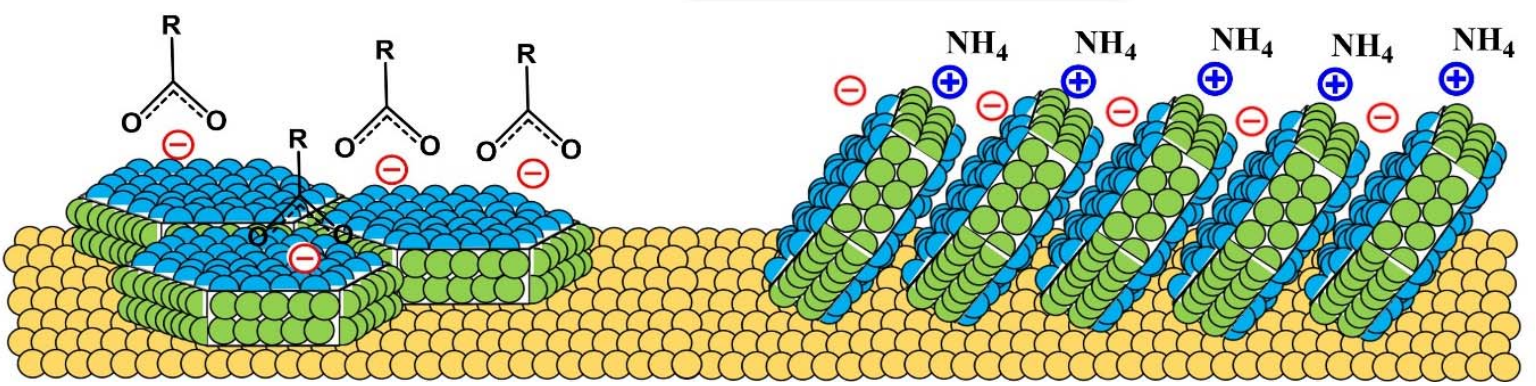

Figure 4. (a) TEM image of high-concentrated $\mathrm{CeF}_{3} \mathrm{NCs}$ synthesized via hydrothermal treatment at $210{ }^{\circ} \mathrm{C}$. Here, the NCs can be observed in a planar (lying flat-red squares) distribution and in a perpendicular (on-edge-blue squares) configuration. In the latter, the interaction between NCs leads to the formation of rod-like assemblies. (b) AFM error signal image enhancing morphological details of an area containing planar and rod-like configurations of $\mathrm{CeF}_{3} \mathrm{NCs}$ deposited by drop-casting on $\mathrm{Au}(111)$ in both orientations, as detailed in Figure 4a. (c) Topographic AFM images of two different areas: (top) area (1300 $\mathrm{nm} \times 1300 \mathrm{~nm}$ ) containing NCs in the planar distribution and (bottom) area $(550 \mathrm{~nm} \times 550 \mathrm{~nm})$ showing the rod-like interaction. Total color scales are $\Delta \mathrm{z}=10 \mathrm{~nm}$. (d) Schematic models for the two NCs configurations found in the TEM and AFM images and the respective observed distributions: (left) planar NCs and (right) rod-like particles. We also indicate the adsorbed ligands and their anionic or cationic character.

Samples consisting of atomically flat $\mathrm{Au}$ (111) films, in which the water dispersion of the NCs was deposited by drop-casting (see Section 2) were measured by AFM over 
areas previously localized by SEM (Figure S9). Figure 4 b shows a general view of the most observed morphological details. As expected from a planar configuration of the platelet-like individual NCs with the $\{0001\}$ facet parallel to the Au substrate surface, randomly distributed particles with heights of 3-10 nm and apparent width from $10 \mathrm{~nm}$ to $60 \mathrm{~nm}$ depending on tip conditions (Figure 4c, top) were found in the vast majority of the inspected areas. Albeit in a small amount all over the surface, some baton-shaped structures (100-200 nm long) were also found (Figure 4c, bottom), ascribed to the rod-like assemblies observed by TEM. Resulting from the attachment of the interacting hexagonal facets, in this configuration, the $\{0001\}$ planes lie out of the surface plane. As observed in Figure $4 \mathrm{~b}$, considerable amounts of rods were found in surface locations close to surface steps. Remarkably, all rods appear oriented along three directions $60^{\circ}$ rotated between each other (Figure $4 b, c$ bottom), indicating the influence of the underlying Au (111) symmetry. Statistical analysis using images taken in different areas indicates that these elongated structures are typically 30-40 nm wide, in agreement with the NC diameter measured in the planar configuration, but they only have an apparent height of $\sim 2-4 \mathrm{~nm}$. Given the excellent vertical resolution of the topographic data (see Au (111) monoatomic steps in Figure S1), this small out-of-plane dimension suggests that the NCs must be tilted with respect to the normal surface (see models of both configurations in Figure 4d).

The contrast in the KPFM maps, arising from the compensation of electrostatic forces at the local level, is commonly attributed to local differences in work function [20], charges, or electrical dipoles [21]. Therefore, KPFM measurements permit obtaining electrostatic surface potential (SP) with local variations at the nanoscale, and they have demonstrated their capability to provide information about surface charges in nanocrystals [22]. Because topography and spatially resolved SP maps are recorded simultaneously, the direct comparison between data in Figure 5 provides a correlation between the NCs organization observed in the topographical image (a) with the corresponding surface potential signal, which results more negative (darker) for the planar NCs and more positive (brighter) for the long assemblies (b). In terms of SP quantification, it is worth mentioning here several facts. On the one hand, the absolute nominal charge of each facet cannot be accurately evaluated by this method because of the unknown extent of (i) charge screening by the metallic support, and/or (ii) variations in charge state with environmental conditions due to their hydrophobic/hydrophilic character. On the other hand, the inclination of the NCs in the rods implies that, in addition to the $\{1 \overline{100}\}$ facets, there is a contribution of the exposed section of the $\{0001\}$ planes (see alternate + and - signs in Figure $4 d$, right), likely provoking altered magnitude of the probed local surface potential. Despite all these considerations, the corresponding SP image (Figure 5b) shows a clear contrast between both types of structures. The surface potential measured on the planar individual NCs (showing \{0001\} planes) is, on average, about $280 \mathrm{mV}$ more negative than for the assemblies of inclined NCs, where the $\{1 \overline{1} 00\}$ planes dominate (see Figure S10). These results nicely confirm the predictions shown in Figure 1 based on all-atom MD simulations.

These findings are in agreement with the expected dual faceted-charge behavior: a positive charge distribution for the rods due to the interaction of the tip with the nonmetal planes (Figure $4 \mathrm{~d}$ right) and a negative charge distribution in the planar NCs due to the exposed hexagonal metallic plane (Figure $4 \mathrm{~d}$ left). As far as we know, this is the first experimental evidence via a microscopy technique of the structural and charge patches in these kinds of systems. 


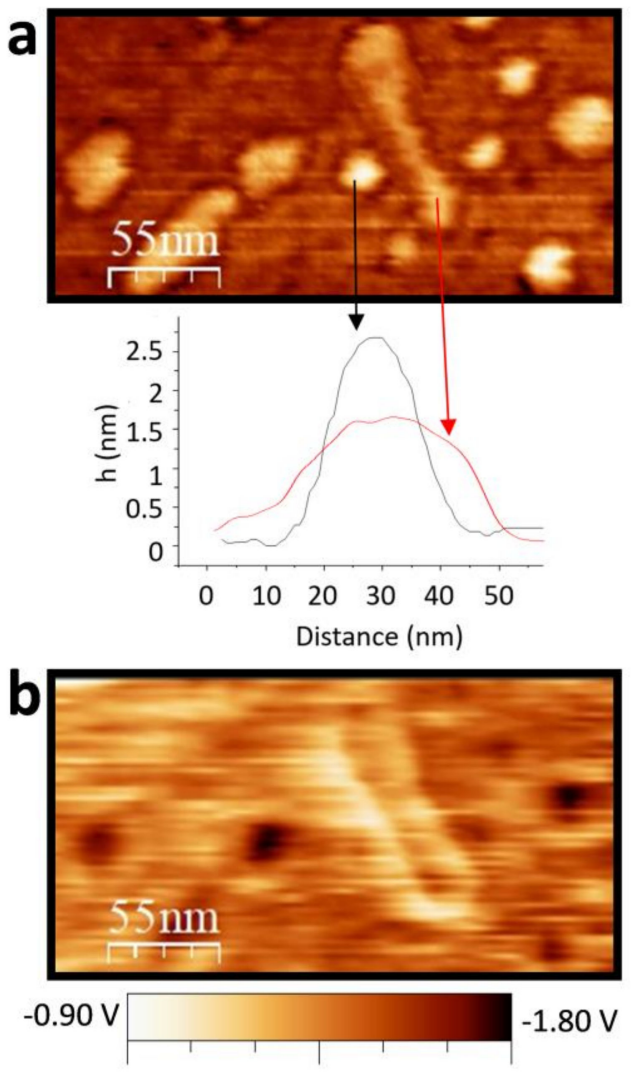

Figure 5. (a) Topographic image and line profiles of typical individual NCs (black) and across the shorter dimension of the rod-like assemblies of inclined NCs. (b) Simultaneous acquired surface potential map by KPFM. Images size is $275 \mathrm{~nm} \times 145 \mathrm{~nm}$ and the color scales are $\Delta \mathrm{z}=10 \mathrm{~nm}$ (a) and $\Delta \mathrm{SP}=900 \mathrm{mV}$ (b) from white to black. The surface potential is lower on the exposed $\{0001\}$ facets than on the surface of the rod (see experimental details and complementary analysis in Figure S10).

\section{Conclusions}

In summary, we reported the tunable-size synthesis of faceted-charge patchy NCs within a range suitable for material science and medical applications (i.e., NCs of a size $>10 \mathrm{~nm}$ are mandatory for reducing toxicity in biological systems) $[23,24]$. Moreover, we provided a microscopic picture of their surface dual properties, hydrophilic/hydrophobic composition, and charged patches. We established a direct comparison at the nanoscale between the electrostatic response of flat-lying single NCs exposing the metal-terminated $\{0001\}$ plane and that of rod-like structures consisting of tilted NCs with most of their exposed surface corresponding to nonmetal $\{1 \overline{1} 00\}$ planes. A notable difference in surface potential as measured by KPFM between the two NC organizations evidenced the dualcharge nature of the patchy system. This work established the foundations for real-space visualization of patchy systems, going one step further in the characterization of single NCs and the identification of their diverse functional sites at the nanoscale. Furthermore, this work demonstrated the suitability of KPFM in identifying the presence of patches of opposite charge in nanoparticles if the size of the particles is suitable for the analysis.

Supplementary Materials: The following are available online at https: / www.mdpi.com/article/ 10.3390/ma15041455/s1: Figure S1: AFM topographic images of Au(111) on mica at two different magnifications. Terraces of several hundred nanometers in width are separated by monoatomic

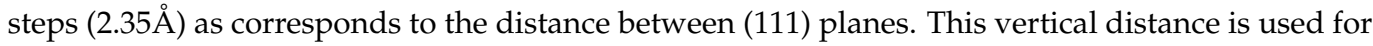
accurate $\mathrm{Z}$ calibration of the images; title; Figure S2: TEM images of LaF3 NCs synthesised at (A) room temperature and (B) room temperature in an ultrasonic batch during 2 h; Figure S3: X-ray diffraction patterns of LaF3 NCs obtained at (A) room temperature and (B) under ultrasonic bath, both of them using the co-precipitation method; Figure S4: TEM images of LaF3 (A) and CeF3 (B) 
patchy NCs via hydrothermal reaction at $210{ }^{\circ} \mathrm{C}$ for $2 \mathrm{~h}$ and LaF3; Figure S5: X-ray diffraction patterns of (A) LaF3 and (B) CeF3 NCs obtained at $210{ }^{\circ} \mathrm{C}$ in a hydrothermal synthesis during 2 h; Figure S6: TEM images of LaF3 (A) and CeF3 (B) patchy NCs via microwave reaction at $200^{\circ} \mathrm{C}$ for $20 \mathrm{~min}$; Figure S7: X-ray diffraction patterns of (A) LaF3 and (B) CeF3 NCs obtained at $200{ }^{\circ} \mathrm{C}$ in a microwave synthesis during 20 minutes; Figure S8: TEM Histograms corresponding to the NCs showed in this paper and summarized in Table 1. (A) LaF3 synthesised at room temperature with and without ultrasonic bath. (B) LaF3 and (C) CeF3 synthesised via high-thermal methods; Figure S9: Scanning Electron microscopy (SEM) image and Electron dispersive X-ray (EDX) of the CeF3 NCs to ensure their presence before AFM technique; Figure S10: (A) Topographical and (B) SP images obtained by KPFM of an area where planar NCs and piles were found with their corresponding numerical plots: (C) hexagonal $\{0001\}$ faces and (D) piles showing the exposed $\{1 \overline{1} 00\}$ planes. The areas of the topographical and SP profiles of NCs (green) and pile (blue) are marked in Figure S10a.

Author Contributions: Conceptualization, S.R., E.B. and C.O.; methodology, J.M.-E. and A.P.-R.; formal analysis, J.M.-E., A.P.-R., R.Y., E.B., J.F., C.O. and S.R.; writing-original draft preparation, J.M.-E. and A.P.-R.; writing-review and editing, J.F., C.O., R.Y. and S.R. All authors read and agreed to the published version of the manuscript.

Funding: This work was supported by the Spanish Government through the "Severo Ochoa" Program for Centers of Excellence in R\&D (CEX2019-000917-S), the COACHSUPENERGY project (MAT201451778-C2-1-R, co-financed by the European Regional Development Fund), and PID2019-110907GB-I00. We also thank the support from the European Union for the ERC Advanced Grant (ULTRASUPERTAPE (ERC-2014-ADG-669504) and IMPACT (ERC-2019-PoC-874964) projects) funded by the European Research Council and from the Catalan Government through 2014-SGR753 and Xarmae.

Institutional Review Board Statement: Not applicable.

Informed Consent Statement: Not applicable.

Data Availability Statement: All data are available in the main manuscript and Supplementary Materials.

Acknowledgments: The authors acknowledge the technical support of Servei de Microscòpia and Servei de Difracció de Raigs-X, all at the UAB.

Conflicts of Interest: J.M.-E. is currently an employee of MDPI journal Nanomaterials.

\section{References}

1. Choueiri, R.M.; Galati, E.; Thérien-Aubin, H.; Klinkova, A.; Larin, E.M.; Querejeta-Fernández, A.; Han, L.; Xin, H.L.; Gang, O.; Zhulina, E.B.; et al. Surface patterning of nanoparticles with polymer patches. Nature 2016, 538, 79-83. [CrossRef]

2. Velev, O.D. Curvature makes a difference. Nat. Nanotechnol. 2013, 8, 620-621. [CrossRef]

3. Zhang, Z.; Glotzer, S.C. Self-Assembly of Patchy Particles. Nano Lett. 2004, 4, 1407-1413. [CrossRef] [PubMed]

4. Fan, W.; Liu, L.; Zhao, H. Co-assembly of Patchy Polymeric Micelles and Protein Molecules. Angew. Chem. Int. Ed. Engl. 2017, 56, 8844-8848. [CrossRef]

5. Pons-Siepermann, I.C.; Glotzer, S.C. Electromechanical Actuator with Controllable Motion, Fast Response Rate, and HighFrequency Resonance Based on Graphene and Polydiacetylene. ACS Nano 2012, 6, 3919-3924. [CrossRef] [PubMed]

6. Harper, E.S.; Van Anders, G.; Glotzer, S.C. The entropic bond in colloidal crystals. Proc. Natl. Acad. Sci. USA 2019, 116, 16703-16710. [CrossRef] [PubMed]

7. Martínez-Esaín, J.; Puig, T.; Obradors, X.; Ros, J.; Yáñez, R.; Faraudo, J.; Ricart, S. Faceted-Charge Patchy LnF 3 Nanocrystals with a Selective Solvent Interaction. Angew. Chem. Int. Ed. Engl. 2018, 5, 14747-14751. [CrossRef] [PubMed]

8. Owen, J. Nanocrystal structure. The coordination chemistry of nanocrystal surfaces. Science 2015, 347, 615-616. [CrossRef]

9. Boles, M.A.; Ling, D.; Hyeon, T.; Talapin, D.V. The surface science of nanocrystals. Nat. Mater. 2016, 15, 141-153. [CrossRef]

10. Humphrey, W.; Dalke, A.; Schulten, K.J. VMD: Visual molecular dynamics. J. Mol. Graph. 1996, 14, 33. [CrossRef]

11. Luo, B.; Smith, J.W.; Wu, Z.; Kim, J.; Ou, Z.; Chen, Q. Polymerization-Like Co-Assembly of Silver Nanoplates and Patchy Spheres. ACS Nano 2017, 11, 7626-7633. [CrossRef]

12. Pothorszky, S.; Zámbó, D.; Szekrényes, D.; Hajnal, Z.; Deák, A. Detecting patchy nanoparticle assembly at the single-particle level. Nanoscale 2017, 9, 10344-10349. [CrossRef]

13. See, E.M.; Peck, C.L.; Santos, W.L.; Robinson, H.D. Light-Directed Patchy Particle Fabrication and Assembly from Isotropic Silver Nanoparticles. Langmuir 2017, 33, 10927-10935. [CrossRef]

14. Cheng, L.; Yang, K.; Li, Y.; Zeng, X.; Shao, M.; Lee, S.; Liu, Z. Multifunctional nanoparticles for upconversion luminescence/MR multimodal imaging and magnetically targeted photothermal therapy. Biomaterials 2012, 33, 2215-2222. [CrossRef] 
15. Sun, Y.; Yu, M.; Liang, S.; Zhang, Y.; Li, C.; Mou, T.; Yang, W.; Zhang, X.; Li, B.; Huang, C.; et al. Fluorine-18 labeled rareearth nanoparticles for positron emission tomography (PET) imaging of sentinel lymph node. Biomaterials 2011, 32, $2999-3007$. [CrossRef]

16. Xia, A.; Gao, Y.; Zhou, J.; Li, C.; Yang, T.; Wu, D.; Wu, L.; Li, F. Core-shell NaYF4:Yb3+, Tm3+ @FexOy nanocrystals for dual-modality T2-enhanced magnetic resonance and NIR-to-NIR upconversion luminescent imaging of small-animal lymphatic node. Biomaterials 2011, 32, 7200-7208. [CrossRef]

17. Xiong, L.; Shen, B.; Behera, D.; Gambhir, S.S.; Chin, F.T.; Rao, J. Synthesis of ligand-functionalized water-soluble[18F]YF3 nanoparticles for PET imaging. Nanoscale 2013, 5, 3253-3256. [CrossRef]

18. You, A.; Be, M.A.Y.; In, I. WSXM: A software for scanning probe microscopy and a tool for nanotechnology. Rev. Sci. Instrum. 2007, 78, 013705.

19. Martínez-Esaín, J.; Faraudo, J.; Puig, T.; Obradors, X.; Ros, J.; Ricart, S.; Yáñez, R. Tunable Self-Assembly of YF3 Nanoparticles by Citrate-Mediated Ionic Bridges. J. Am. Chem. Soc. 2018, 140, 2127-2134. [CrossRef]

20. Sadewasser, S.; Glatzel, T.; Rusu, M.; Lux-Steiner, M.C. High-resolution work function imaging of single grains of semiconductor surfaces. Appl. Phys. Lett. 2012, 80, 2979. [CrossRef]

21. Barth, C.; Henry, C.R. Gold nanoclusters on alkali halide surfaces: Charging and tunneling. Appl. Phys. Lett. 2011, 89, 252119. [CrossRef]

22. Geisel, K.; Rudov, A.A.; Potemkin, I.I.; Richtering, W. Hollow and Core-Shell Microgels at Oil-Water Interfaces: Spreading of Soft Particles Reduces the Compressibility of the Monolayer. Langmuir 2015, 31, 13145-13154. [CrossRef] [PubMed]

23. Albanese, A.; Tang, P.S.; Chan, W.C.W. The effect of nanoparticle size, shape, and surface chemistry on biological systems. Annu. Rev. Biomed. Eng. 2012, 14, 1-16. [CrossRef] [PubMed]

24. Nel, A.; Xia, T.; Mädler, L.; Li, N. Toxic Potential of Materials at the Nanolevel. Science 2006, 311, 622-627. [CrossRef] [PubMed] 